\title{
LIQUISOLID COMPACT TECHNIQUES: A REVIEW
}

\author{
MANISHA ROKADE*, PRADNYA KHANDAGALE, DIPTI PHADTARE
}

\author{
R. G. Sapkal Institute of Pharmacy, Anjaneri Nashik \\ Email: rokademanisha3131@gmail.com
}

Received: 21 Apr 2018, Revised and Accepted: 10 Jun 2018

\begin{abstract}
The poor dissolution rate of water-insoluble drugs is still a substantial problem confronting the pharmaceutical industry. There are several methods used to increase the solubility of drugs, of those liquid-solid compact technique is a new and promising addition towards such a novel aim, that the solubility of the insoluble drug moiety is increased by the aid of non-volatile solvents and hence increasing the dissolution and bioavailability. Oral drug administration has been one of the most convenient and widely accepted routes of delivery for most of the therapeutic agents. It is one of the most extensively used routes of drug administration because of its obvious advantages of ease of administration, improved patient compliance, and convenience. The enhancement of oral bioavailability of poorly water-soluble drugs remains one of the most challenging aspects of drug development. A more recent technique, "powdered solution technology" or "Liquisolid technology", has been applied to prepare water-insoluble drugs into rapid-release solid dosage forms. The limited solubility of drugs is a challenging issue for the industry, during the development of the ideal solid dosage form unit. The technique is based upon the dissolving the insoluble drug in the nonvolatile solvent and admixture of drug loaded solutions with appropriate carrier and coating materials to convert into acceptably flowing and compressible powders.
\end{abstract}

Keywords: Liquisolid System, Liquid load factor, Carrier material, Coating material, Non-volatile solvents

(C) 2018 The Authors. Published by Innovare Academic Sciences Pvt Ltd. This is an open access article under the CC BY license (http://creativecommons.org/licenses/by/4.0/)

DOI: http://dx.doi.org/10.22159/ijcpr.2018v10i4.28465

\section{INTRODUCTION}

Bioavailability is the key determinant of a drug for its therapeutic effectiveness, which in turn depends upon the solubility of that drug in gastrointestinal fluid. Solubility is one of the important parameters to achieve the desired concentration of drug in systemic circulation for pharmacological response [1]. Poorly water-soluble drugs will be inherently released at a slow rate owing to their limited solubility within the GI contents. The dissolution rate is often the ratedetermining step in the drug absorption. The challenge for poorly water-soluble drugs is to enhance the rate of dissolution. This in turn subsequently improves absorption and bioavailability. Formulation methods targeted at dissolution enhancement of poorly soluble substances are continuously introduced. Bioavailability of poorly water-soluble drugs is limited by their solubility and dissolution rate. Several studies have been carried out to increase the dissolution rate of drugs by decreasing the particle size, by creating nano and microparticles. However, the fine drug particles have a high tendency to agglomerate due to van-der Waals attraction or hydrophobicity, which result in a decrease in surface area over time. Another way of increasing the dissolution rate is the adsorption of the drug onto a high-surface-area carrier. In this technique, the drug is dissolved in an organic solvent followed by soaking of the solution by a high-surfacearea carrier such as silica. Here, agglomeration of the drug particles is prevented due to the binding of the drug to the carrier. However, due to the presence of the residual solvent in the drug formulation, it is disadvantageous to use toxic solvents [2]. Over the years, various techniques have been employed to enhance the dissolution profile and, in turn, the absorption efficiency and bioavailability of waterinsoluble drugs and/or liquid lipophilic medications.

Nowadays, the synthesis of poorly soluble drugs increasing steadily. Therapeutic effectiveness of a drug depends upon the bioavailability which is dependent on the solubility and dissolution rate of drug molecules. Solubility is one of the important parameters to achieve the desired concentration of drug in systemic circulation for pharmacological response to be shown. The drugs which are poorly water soluble will be inherently released at a slow rate owing to their limited solubility within the GI contents. The dissolution rate is often the rate-determining step in the drug absorption. The challenge for these drugs is to enhance the rate of dissolution or solubility. This in turn subsequently improves absorption and bioavailability. Formulation methods targeted at dissolution enhancement of poorly soluble substances are continuously introduced [3]. Liquisolid system refers to formulations formed by conversion of liquid drugs, a drug suspension or drug solution in non-volatile solvents into non-adherent, free-flowing and compressible powder mixtures by blending the solution or suspension with selected carriers and coating materials. As large proportions of new drug candidates have a poor aqueous solubility, various formulation strategies were reported to overcome such a problem. Among these techniques is complexation with cyclodextrins, micronization, solid dispersion, co-precipitation and recently, the technique of 'liquisolid compacts'. Several studies have shown that the liquisolid technique is a promising method for promoting the dissolution rate of poorly water-soluble drugs [4].

The concept of "liquisolid tablets" was evolved from "powdered solution technology" that can be used to formulate "liquid medication". The term "liquid medication" refers to solid drugs dispersed in suitable non-volatile liquid vehicles. By simple mixing of such "liquid medication" with selected carriers and coating materials, dry-looking, non-adherent, free-flowing and readily compatible powder admixtures can be produced. Spireas and Bolton suggested that particles possess porous surface with high absorption properties may be used as the carrier material such as cellulose, starch and lactose. Increasing moisture content of carrier's results in decreased powder flowability. Coating material is required to cover the surface and so maintain the powder flowability. Accordingly, the coating material should be a very fine and highly adsorptive silica powders [5]. Liquisolid compacts are acceptably flowing and compressible powdered forms of liquid medications. The term liquid medication implies oily, liquid drugs and solutions or suspensions of water-insoluble solid drugs carried in suitable non-volatile solvent systems termed the liquid vehicles. Using this new formulation technique, a liquid medication may be converted into a dry-looking, non-adherent, free-flowing, and readily compressible powder by a simple blending with selected powder excipients referred to as the carrier and coating materials Various grades of cellulose, starch, lactose, and so on, may be used as the carriers, whereas very fine-particle-size silica powders may be used as the coating [or covering] materials. In liquisolid compacts, even though the drug is in a tabletted or encapsulated dosage form, it is held in a solubilized liquid state, which consequently contributes to increased drug wetting properties, thereby enhancing drug 
dissolution. Another advantage of liquisolid systems is that their production cost is lower than that of soft gelatin capsules because the production of liquisolid systems is similar to that of conventional tablets [6].

\section{Historical development}

Historically, liquisolid compacts are descendants of 'powdered solutions', an older technique which was based on the conversion of a solution of a drug in a nonvolatile solvent into a dry-looking, nonadherent powder by mainly adsorbing the liquid onto silicas of large specific surfaces. Such preparations, however, have been investigated for their dissolution profiles while being in a powder dispersion form and not as compressed entities, simply because they could not be compressed into tablets. In later studies on powdered solutions, compression enhancers such as microcrystalline cellulose were added in such dispersions in order to increase the compressibility of the systems. In these studies, however, large quantities of silicas were still being used, and the flow and compression properties of the products were never validated and standardized to industrial specifications and requirements. Liquisolid compacts, on the other hand, are acceptably flowing and compressible powdered forms of liquid medications, and have industrial application. In addition, the term 'liquid medication' does not only imply drug solutions, as in powdered solutions, but also drug suspensions, emulsions, or liquid oily drugs. Therefore, in contrast to 'powdered solutions', the term 'liquisolid compacts' is more general and it may encompass four different formulation systems namely,

\section{Powdered drug solutions}

\section{Powdered drug suspensions}

\section{Powdered drug emulsions}

\section{Powdered liquid drugs}

Furthermore, the earlier term 'powdered solutions' seems to be inadequate even in describing the original systems, since it has not been proven that the drug remains in solution in the liquid vehicle after its deposition on the extremely large powder surfaces of silica used. The new 'liquisolid' technique may be applied to formulate liquid medications [i.e., oily liquid drugs and solutions, suspensions or emulsions of water-insoluble solid drugs carried in nonvolatile liquid vehicles] into powders suitable for tableting or encapsulation. Simple blending of such liquid medications with calculated quantities of a powder substrate consisting of certain excipients referred to as the carrier and coating powder materials can yield dry-looking, nonadherent, free-flowing, and readily compressible powders [7].

\section{Concept}

When the drug dissolved in the liquid vehicle is incorporated into a carrier material which has a porous surface and closely matted fibers in its interior as cellulose, both absorption and adsorption take place; i.e., the liquid initially absorbed in the interior of the particles is captured by its internal structure, and after the saturation of this process, adsorption of the liquid onto the internal and external surfaces of the porous carrier particles occur. Then, the coating material having high adsorptive properties and large specific surface area gives the liquisolid system the desirable flow characteristics [8]. In liquisolid systems, the drug is already in solution in a liquid vehicle, while at the same time, it is carried by the powder particles [microcrystalline cellulose and silica]. Thus, due to significantly increased wetting properties and surface area of drug available for dissolution, liquisolid compacts of water-insoluble substances may be expected to display enhanced drug release characteristics and consequently, improved oral bioavailability. Since the dissolution of a non-polar drug is often the rate-limiting step in gastrointestinal absorption, the better bioavailability of an orally administered water-insoluble drug is achieved when the drug is already in solution, thereby displaying enhanced dissolution rates. That is why soft gelatin elastic capsules containing solutions of such medications demonstrate higher bioavailability when compared to conventional oral solid dosage forms. A similar principle underlies the mechanism of drug delivery from liquisolid compacts and is chiefly responsible for the improved dissolution profiles exhibited by these preparations. The wettability of the compacts by the dissolution media is one of the proposed mechanisms for explaining the enhanced dissolution rate from the liquisolid compacts. The nonvolatile solvent present in the liquisolid system facilitates wetting of drug particles by decreasing interfacial tension between dissolution medium and tablet surface $[9,10]$.

Fig. 1 shows a lower contact angle of liquisolid compacts than the conventional tablets and thus improved wettability.

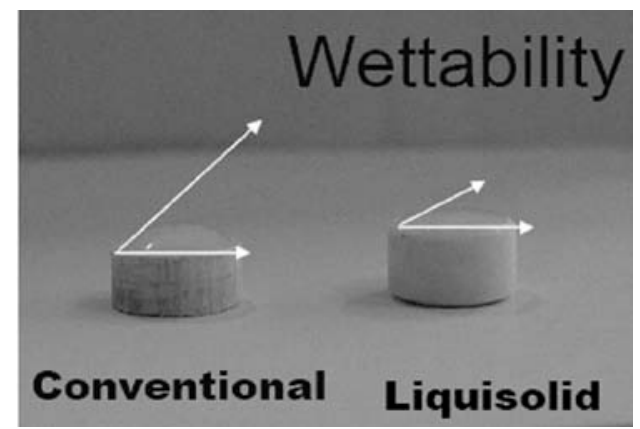

Fig. 1: Comparison of wettability between conventional tablet and liquisolid compacts

\section{Components}

The major formulation components of liquisolid compacts are:

\section{Carrier material}

These are compression-enhancing, relatively large, preferably porous particles possessing a sufficient absorption property which contributes in liquid absorption.

E. g. various grades of cellulose, starch [9], lactose [9], sorbitol [10] etc.

\section{Coating material}

These are flow-enhancing, very fine $[10 \mathrm{~nm}$ to $5,000 \mathrm{~nm}$ in diameter], highly adsorptive coating particles [e. g., silica of various grades like Cab-0-Sil M5, Aerosil 200, Syloid 244FP etc.] contributes in covering the wet carrier particles and displaying a dry-looking powder by adsorbing any excess liquid [11-13].

\section{Non-volatile solvents}

Inert, high boiling point, preferably water-miscible and not highly viscous organic solvent systems e. g., propylene glycol, liquid polyethene glycols, polysorbates, glycerin, N, N-dimethylacetamide, fixed oils, etc. are most suitable as vehicles.

\section{Disintegrants}

Most commonly used disintegrant is sodium starch glycolate [Explotab13, Pumogel, etc.]

\section{Lubricant}

Most commonly used lubricant is magnesium state.

\section{Classification of liquisolid systems}

A. Based on the type of liquid medication contained Therein, liquisolid systems may be classified into three subgroups:

1. Powdered drug solutions

2. Powdered drug suspensions

\section{Powdered liquid drugs}

The first two may be produced from the conversion of drug solutions or [e. g. prednisolone solution in propylene glycol] or drug suspensions [e. g. gemfibrozil suspension in Polysorbate 80], and the 
latter from the formulation of liquid drugs [e. g. clofibrate, liquid vitamins, etc.], into liquisolid systems. Since non-volatile solvents are used to prepare the drug solution or suspension, the liquid vehicle does not evaporate and thus, the drug is carried within the liquid system which in turn is dispersed throughout the final product.

B. Based on the formulation technique used, liquisolid systems may be classified into two categories:

\section{Liquisolid compacts}

\section{Liquisolid microsystems.}

Liquisolid compacts are prepared using the previously outlined method to produce tablets or capsules, whereas the liquisolid microsystems are based on a new concept which employs similar methodology combined with the inclusion of an additive, e. g. Polyvinylpyrrolidone [PVP], in the liquid medication which is incorporated into the carrier and coating materials to produce an acceptably flowing admixture for encapsulation. The advantage stemming from this new technique is that the resulting unit size of liquisolid microsystems may be as much as five times less than that of liquisolid compacts [14].

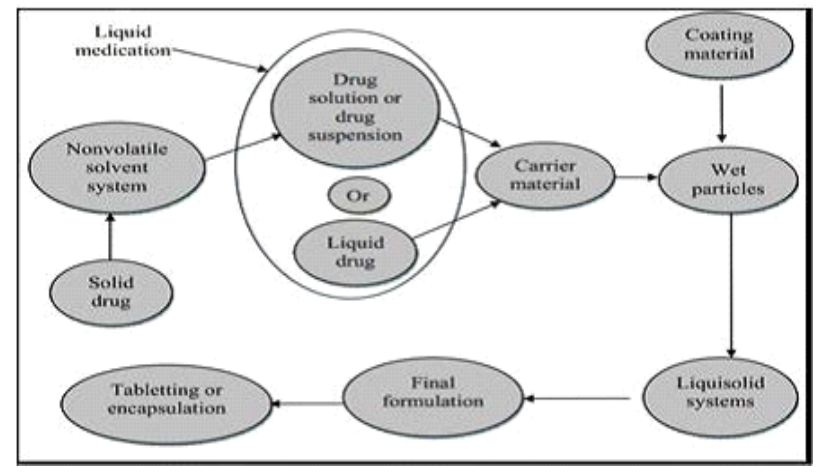

Fig. 2: Steps involved in the preparation of liquisolid systems

\section{General procedure for liquisolid tablets}

The liquisolid tablet preparation method involves, first a mathematically calculated amount of pure drug weighed and dissolved in the suitable amount of solvent in a molecularly dispersed state. For attaining good flow properties trial and error methods were used i.e. changing the carrier: coating material ratio from 50:1 to 5:1 ratios according to new mathematical model expressions proposed by Liao [15]. This liquid medication is poured on the suitable amount of carrier material. The liquid medication is absorbed into the carrier material internally and externally and then a suitable disintegrant was added to this material. Finally, coating material was added for dry looking, adherent to the carrier material for achieving good compression properties. Liquid medication is incorporated into carrier material which has a porous surface and closely matted fibers in its interior as cellulose [16]. Both absorption and adsorption take place, i.e. the liquid absorbed into the interior of the particles is captured by its internal structure and after saturation of this process, adsorption of the liquid onto the internal and external surface of the porous carrier particles occurs. Excipients possessing a fine and highly adsorptive particles such as various types of amorphous silicon dioxide (silica) are most suitable for this step. Before compression or encapsulation, various ingredients such as lubricants disintegrants or Polymers, and binders (as shown in fig. 3), may be mixed with the finished liquisolid systems to produce liquisolid compacts in the dosage form of tablets or capsules [17-19].

Examples of some drugs that can be incorporated into liquisolid systems
1. Chlorpheniramine
2. Digoxin
3. Nifedipine

\author{
4. Clofibrate \\ 5. Gemfibrozil \\ 6. Etoposide \\ 7. Carbamazepine \\ 8. Hydrochlorothiazide \\ 9. Methyclothiazide \\ 10. Spironolactone \\ 11. Hydrocortisone \\ 12. Piroxicam \\ 13. Indomethacin \\ 14. Ibuprofen
}

Determination of drug in different non-volatile solvents

These are carried by preparing saturated solutions of the drug in non-volatile solvents and analyzing them spectrophotometrically. Saturated solutions are prepared by adding an excess of the drug to vehicles and shaking them on a shaker for the specific time period under steady vibration. After this, the solutions are filtered and analyzed spectrophotometrically.

\section{Advantages of liquisolid tablets [20]}

Liquisolid tables have many advantages. Few of them are:

- Liquisolid systems are low-cost formulations than soft gelatin capsules.

- Production of them is similar to that of conventional tablets.

- Drug release can be modified using suitable formulation ingredients

- The drug can be molecularly dispersed in the formulation.

- The capability of industrial production is also possible.

- Enhanced bioavailability can be obtained as compared to conventional tablets.

\section{Applications of liquisolid techniques [20]}

\section{Solubility and dissolution improvement [21]}

This technique was successfully applied for low dose water-insoluble drugs. However, formulation of the high dose insoluble drugs as liquisolid tablets is one of the limitations of the liquisolid technique. In fact, when the therapeutic dose of the drug is more than $50 \mathrm{mg}$ dissolution enhancement in the presence of low levels of hydrophilic carrier and coating material is not significant. But by adding some materials such as polyvinylpyrrolidone (PVP) to liquid medication (microsystems), it would be possible to produce dry powder formulations containing a liquid with a high concentration of the drug. By adding such materials to the liquid medication, low amount of carrier is required to obtain a dry powder with free flowability and good compatibility [22].

\section{Flowability and compressibility}

Liquisolid compacts possess acceptable flowability and compressibility properties. They are prepared by simple blending with selected powder excipients referred to as the carriers and the coating materials. Many grades of cellulose, starch, lactose, etc. can be used as carriers, whereas silicas of very fine particle size can be used as coating materials. In order to have acceptable flowability and compactibility for liquisolid powder formulation, high levels of carrier and coating materials should be added and that in turn will increase the weight of each tablet above 1 gm which is very difficult to swallow. Therefore, in practice, it is impossible with the conventional method to convert high dose drugs to a liquisolid tablet with the tablet weight of less than $1 \mathrm{gm}$. In such systems, the drug existed in a molecular state of subdivision and systems were freeflowing, on-adherent, dry looking powders. In further studies, compression enhancers were added to these resulted in a significant "Liquid Squeezing Out" phenomenon. 


\section{Bioavailability improvement}

In the liquisolid and powdered solution systems the drug might be in a solid dosage form, it is held within the powder substrate in solution, or in a solubilized, almost molecularly dispersed state. Therefore, due to their significantly increased wetting properties and the surface of drug available for dissolution, liquisolid compacts of water-insoluble substances may be expected to display enhanced drug release properties, and consequently, improved bioavailability.

\section{Limitations}

1) Not applicable for the formulation of high dose insoluble drugs.

2) If more amount of carrier is added to produce free flowing powder, the tablet weight increases to more than one gram which is difficult to swallow.

3) Acceptable compression properties may not be achieved since during compression liquid drug may be squeezed out of the liquisolid tablet resulting in tablets of unsatisfactory hardness.

4) Introduction of this method on an industrial scale and to overcome the problems of mixing small quantities of viscous liquid solutions onto large amounts of carrier material may not be feasible [23]

\section{Evaluation of liquisolid granules}

\section{Flow behaviour}

The flowability of a powder is of critical importance in the production of pharmaceutical dosage forms in order to reduce high dose variations [23]. Angle of repose, Carr's index and Hausner's ratio were used in order to ensure the flow properties of the liquisolid systems.

\section{Angle of repose [24]}

This is the maximum angle possible between the surface of a pile of powder and the horizontal plane.10 gm of powder was allowed to flow by funnel from $4 \mathrm{~cm}$ of height from the base. The height of pile and diameter of the base was measured and calculate the angle of repose by the following the formula.

$\tan \theta=\mathrm{h} / \mathrm{r}$

$\theta=\tan -1 \mathrm{~h} / \mathrm{r}$

Where $=$ angle of repose,

$\mathrm{h}=$ Height of the heap,

$r=$ Radius of the heap.

\section{Bulk density [24]}

An accurately weighed quantity of powder, which was previously passed through sieve \# 40 [USP] and carefully poured into a graduated cylinder. Then after pouring the powder into the graduated cylinder, the powder bed was made uniform without disturbing. Then the volume was measured directly from the graduation marks on the cylinder as ml. The volume measured was called as the bulk volume and the bulk density is calculated by following formula;

Bulk density $=$ Weight of powder/Bulk volume.

\section{Tapped density [24]}

After measuring the bulk volume the same measuring cylinder was set into tap density apparatus. The tap density apparatus was set to 300 taps drop per minute and operated for 500 taps. Volume was noted as [Va] and again tapped for 750 times and volume was noted as [Vb].

If the difference between $\mathrm{Va}$ and $\mathrm{Vb}$ not greater than $2 \%$ then $\mathrm{Vb}$ is to consider as final tapped volume. The tapped density is calculated by the following formula

Tapped density $=$ Weight of powder/Tapped Volume.

\section{Carr's index [compressibility index] [25]}

It is one of the most important parameters to characteristic the nature of powders and granules. It can be calculated from the following equation-
Carr's index $=$ Tapped density-Bulk density/Tapped density X 100.

\section{Hausner's ratio [26]}

Hausner's ratio is an important character to determine the flow property of powder and granules. This can be calculated by the following formula-

Hausner's ratio $=$ Tapped density $/$ Bulk density.

\section{Evaluation of liquisolid tablets $[27,28]$}

\section{Weight variation}

Weight variation was measured by weighing 20 Tablets and average weight was found and percentage weight variation of the individual tablet should fall within specified limits in terms of percentage deviation from the mean.

\section{Thickness}

The thickness of the tablet was measured by vernier caliper.

\section{Hardness}

It is a measure of the mechanical strength of a tablet using a hardness tester [Monsanto hardness tester]. The mechanical strength of a tablet is associated with the resistance of a tablet to fracture or attrition.

\section{Friability}

It was determined using Roche friabilator, the percentage loss in tablet weight before and after 100 revolutions of tablets was calculated and taken as a measure for friability.

\section{Disintegration time}

The time necessary to disintegrate 3 tablets of each tablet formulation was determined using a disintegration tester.

\section{In vitro dissolution studies}

It is carried out as given in particular monograph of the drugs tablet formulation.

\section{CONCLUSION}

The addition of disintegrants may further accelerate drug release from liquisolid compacts. The liquisolid technology may also be used for the preparation of sustained release formulations with zero order release pattern. Thus, a constant plasma level will be reached, which is maintained throughout the dosing interval. For sustained release liquisolid compacts, the selection and the concentration of the excipients such as a liquid vehicle, retarding agent (matrix forming material) as well as carrier and coating material play an important role.

\section{AUTHORS CONTRIBUTIONS}

All the author have contributed equally

\section{CONFLICT OF INTERESTS}

Declared none

\section{REFERENCES}

1. Mohamed Hassan G. Dehghan and mohammad jafar-improving dissolution of meloxicam using solid dispersions. Iranian J Pharm Res 2004;5:231-8.

2. Ellsworth AJ, Witt DM, Dug dale DC. Medical drug reference. Elsevier science, Missouri; 2003. p. 610-2.

3. Nagabandi VK, Ramarao T, Jayaveera KN. LIQUISOLID compacts: a novel approach to enhance bioavailability of poorly soluble drugs. Int J Pharm Biol Sci 2011;1:89-102.

4. Chuahan PV, Patel HK, Patel BA, Patel KN, Patel PA. Liquisolid technique for enhancement of dissolution rate of ibuprofen. Int J Pharm Res Scholars 2012;1:268-80.

5. Ngiik T, Elkordy A. Effects of liquisolid formulations on dissolution of naproxen. Eur J Pharm Biopharma 2009;73:373-84.

6. Kulkarni AS, Gaja JB. Formulation and evaluation of liquisolid compacts of diclofenac sodium. PDA J Pharm Sci Technol 2010;64:222-32. 
7. El-Say KM, Samy AM, Fetouh MI. Formulation and evaluation of rofecoxib liquisolid tablets. Int J Pharm Sci Rev Res 2010;3:135-42.

8. Fahmy RH, Kassem MA. Enhancement of famotidine dissolution rate through liquisolid tablet formulation: in vitro and in vivo evaluation. Eur J Pharm Biopharm 2008;69:993-1003.

9. Javadzadeh Y, Navimipour B, Nokhodchi A. Liquisolid technique for dissolution rate enhancement of a high dose water-insoluble drug [carbamazepine]. Int J Pharm 2007;341: 26-34.

10. Javadzadeh Y, Siahi MR, Asnaashri S. An investigation of physicochemical properties of piroxicam liquisolid compacts. Pharm Dev Tech 2007;12:337-4.

11. Spiras S. Liquisolid systems and methods for preparing same, United States patent 2002;6:423, 339.

12. Spiras S, Bolton SM. Liquisolid systems and methods for preparing same, United States Patent 2000;6:96, 337.

13. Spiras S, Wang T, Grover R. Effect of powder substrate on dissolution properties of methyclothiazide Liquisolid Compacts. Drug Dev Ind Pharm 1999;25:63-168.

14. Banker GS, Anderson NL. Tablets. In: The theory and practice of industrial pharmacy. Lachman L, Liberman HA, Kanig JL. edn. 3rd. Varghese Publishing House, Bombay, India; 1987. p. 293345.

15. Nazzal S, Khan MA. Controlled release of a self-emulsifying formulation from a tablet dosage form: Stability assessment and optimization of some processing parameters. Int J Pharm 2006;315:110-21.

16. Grover R, Spireas S, Wang T. Effect of powder substrate on the dissolution properties of Methchrothiazide liquisolid compacts. Drug Dev Ind Pharm 1999;25:163-8.
17. Bolton S, Spireas S. Sustained-release liquisolid compacts. In: $25^{\text {th }}$ International Symposium on Controlled Release of Bioactive Materials., Nevada, USA; 1998. p. 138-9.

18. Staniforth J. Powder flow. In: M Aulton. Pharmaceutics: the Science of Dosage Form Design, Edinburgh; 2002. p. 197-210.

19. Wells J. Pharmaceutical Preformulation: The physicochemical properties of drug substances. In: Aulton M. Pharmaceutics: the Science of Dosage Form Design, Edinburgh; 2002. p. 114-38.

20. Nokhodchi A. The effect of type and concentration of vehicles on the dissolution rate of a poorly soluble drug (indomethacin) from liquisolid compacts. J Pharm Sci 2005;8:18-25.

21. Amrit B Karmarkar, Indrajeet D Gonjari, Avinash H Hosmani, Pandurang N Dhabale, Satish B Bhise. Liquisolid tablets: a novel approach for drug delivery. Int J Health Res 2009;2:45-50.

22. PM Sabale. Liquisolid technique for enchancement of dissolution properties of Fenofibrate. Int J Pharma Sci Res 2012;3:1481-6.

23. Sambasiva RA, Naga AT. Liquisolid technology: an overview. Int J Res Pharm Biomed Sci 2011;2:401-9.

24. Subhramanyam CVS. Textbook of physical pharmaceutics. $2^{\text {nd }}$ edition. Vallabh Prakashan. New Delhi; 2001.

25. Vajir S. Enhancement of dissolution rate of poorly watersoluble diclofenac sodium by liquisolid technique. Int J Pharm Chem Sci 2012;1:989-1000.

26. Patric JS. Martin's physical pharmacy and pharmaceutical sciences. 5th ed. Lippincott Williams and Wilkins; 2003.

27. Chuahan PV, Patel HK, Patel BA, Patel KN, Patel PA. Liquisolid technique for enhancement of dissolution rate of ibuprofen. Int J Pharm Res Scholars 2012;1:268-80.

28. Patric JS. Martin's physical pharmacy and pharmaceutical sciences. 5th ed. Lippincott Williams and Wilkins; 2003. 